\title{
Syntheses and chemistry of Tris(2-pyridyl)phosphine complexes of Group VI transition metals. X-ray structural studies of the molybdenum complexes
}

\author{
Cheng-Yi Kuo a, Yaw-Shyan Fuh a, Jay-Yun Shiue ${ }^{\text {a }}$, S. Joyce $\mathrm{Yu}^{\text {a,*, }}{ }^{\text {, }}$ \\ Gene-Hsiang Lee ${ }^{\mathrm{b}}$, Shie-Ming Peng ${ }^{\mathrm{b}}$ \\ a Department of Chemistry, National Chung Cheng University, Ming Hsiung, Chia Yi 621, Taiwan, ROC \\ ${ }^{\mathrm{b}}$ Department of Chemistry, National Taiwan University, Taipei 106, Taiwan, ROC
}

Received 11 May 1999; received in revised form 28 June 1999

\begin{abstract}
Treatment of $\mathrm{P}(2-\mathrm{py})_{3}$ with $\mathrm{Mo}(\mathrm{CO})_{6}$ or $\mathrm{M}(\mathrm{CO})_{3}\left(\mathrm{CH}_{3} \mathrm{CN}\right)_{3}(\mathrm{M}=\mathrm{Cr}, \mathrm{W})$ in $\mathrm{CH}_{3} \mathrm{CN}$ affords compounds of the type, $\mathrm{P}(2-\mathrm{py})_{3} \mathrm{M}(\mathrm{CO})_{3}(\mathbf{1 a}, \mathrm{M}=\mathrm{Cr} ; \mathbf{1 b}, \mathrm{M}=\mathrm{Mo} ; \mathbf{1 c}, \mathrm{M}=\mathrm{W})$. Complex $\mathrm{P}(2-\mathrm{py})_{3} \mathrm{Mo}(\mathrm{CO})_{3} \cdot \mathrm{CH}_{2} \mathrm{Cl}_{2}$ was structurally characterized, and its ORTEP drawing shows an almost perfect octahedral arrangement around the Mo center, and the $\mathrm{P}(2 \text {-py })_{3}$ ligand occupying the facial position of the octahedron through the coordination of three pyridyl nitrogen atoms. Substitution of CO ligands can take place when treating compounds $\mathbf{1 b}$ and $\mathbf{1 c}$ with one or two equiv. of $\mathrm{NOBF}_{4}$ in $\mathrm{CH}_{3} \mathrm{NO}_{2}$ solvent to afford $\left[\mathrm{P}(2-\mathrm{py})_{3} \mathrm{M}(\mathrm{CO})_{3}\right.$ $\left.n(\mathrm{NO})_{n}\right]\left(\mathrm{BF}_{4}\right)_{n}(\mathbf{2 a}, \mathrm{M}=\mathrm{Mo}, n=1 ; \mathbf{2} \mathbf{b}, \mathrm{M}=\mathrm{W}, n=1 ; \mathbf{3 a}, \mathrm{M}=\mathrm{Mo}, n=2 ; \mathbf{3 b}, \mathbf{M}=\mathbf{W}, n=2)$. These singly and doubly charged cationic species have been characterized by ${ }^{1} \mathrm{H}-,{ }^{13} \mathrm{C}-,{ }^{31} \mathrm{P}-,{ }^{19} \mathrm{~F}-\mathrm{NMR}$ and IR spectroscopy, as well as elemental analysis. The $\mathrm{CO}$ lability of $\mathbf{3 a}$ and $\mathbf{3 b}$ generated the 16-electron species, $\left[\mathrm{P}(2-\mathrm{py})_{3} \mathrm{M}(\mathrm{NO})_{2}\right]^{2+}$, which binds to most Lewis basic donor ligands to give complexes of the type $\left[\mathrm{P}(2-\mathrm{py})_{3} \mathrm{M}(\mathrm{L})(\mathrm{NO})_{2}\right]\left(\mathrm{BF}_{4}\right)_{2} \quad(\mathrm{~L}=$ nitriles, aldehydes). A single crystal of $[\mathrm{P}(2-$ py $\left.)_{3} \mathrm{M}\left(\mathrm{CH}_{3} \mathrm{CH}_{2} \mathrm{CN}\right)(\mathrm{NO})_{2}\right]\left(\mathrm{BF}_{4}\right)_{2} \cdot \mathrm{CH}_{3} \mathrm{NO}_{2}$ was also isolated and structurally characterized. The crystallographic analysis indicated an octahedral arrangement about the Mo metal center. The ${ }^{1} \mathrm{H}-\mathrm{NMR}$ downfield shift of the metal-coordinated crotonaldehyde in compounds $\mathbf{4 c}$ and $\mathbf{5 c}$ is used as a qualitative measurement of relative Lewis acidity, and the acid strength of $\left[\mathrm{P}(2-\mathrm{py})_{3} \mathrm{M}(\mathrm{NO})_{2}\right]^{2+}$ is comparable to that of $\mathrm{TiCl}_{4}$ when $\mathrm{M}=\mathrm{Mo}$ and to $\mathrm{BF}_{3}$ and $\mathrm{AlCl}_{3}$ when $\mathrm{M}=\mathrm{W}$. The Diels-Alder reactions between cyclohexadienes and methyl vinyl ketone were catalyzed by $0.3 \mathrm{~mol} \%$ of $\left[\mathrm{P}(2-\mathrm{py})_{3} \mathrm{~W}(\mathrm{CO})(\mathrm{NO})_{2}\right]\left(\mathrm{BF}_{4}\right)_{2}(3 \mathbf{b})$ to afford an average $94 \%$ conversion and 83\% isolated yield. (C) 1999 Elsevier Science S.A. All rights reserved.
\end{abstract}

Keywords: Group VI transition metals; Molybdenum; X-ray

\section{Introduction}

The synthesis of Tris(2-pyridyl)phosphine (P(2-py) ${ }_{3}$, 2-py $=2$-pyridyl) was first reported [1] in 1944 by Davies and Mann as part of a study on the optical resolution of tertiary phosphines. Yet the complexation of $\mathrm{P}(2-\mathrm{py})_{3}$ with metal ions was not reported until 1970 [2]. There has been an increasing usage of $\mathrm{P}(2-\mathrm{py})_{3}$ in inorganic and organometallic chemistry primarily because of its interesting structural feature retaining both phosphorus and nitrogen atoms with versatile coordi-

* Corresponding author. Fax: + 886-5-2721040.

E-mail address: chejyy@ccunix.ccu.edu.tw (S. Joyce Yu) nation possibility. There are four coordination modes found for complexes containing $\mathrm{P}(2-\text { py })_{3}$, namely, Pmonodentate $[3,4], \eta^{2}-P, N$-chelate $[5,6 \mathrm{a}], \mu-P, N$-bridge [7] and $\eta^{3}-N, N^{\prime}, N^{\prime \prime}$-chelate [8]. Thus, application of $\mathrm{P}(2-p y)_{3}$ ligations affords numerous novel and useful complexes. Apart from its characteristics in coordination chemistry, the catalysis aspects of $\mathrm{P}(2-\mathrm{py})_{3}$ for the reactions employing triphenylphosphine have been recently explored [6]. We have focused our interest on the $\eta^{3}-N, N^{\prime}, N^{\prime \prime}-\mathrm{P}(2-p y)_{3}$ ligation, where the $\mathrm{P}(2-\mathrm{py})_{3}$ ligand binds to group VI transition metals at facial position of an octahedron via three pyridyl nitrogen atoms. The $\eta^{3}-N, N^{\prime}, N^{\prime \prime}$-chelate has been mostly observed as a $\pi$-acceptor ligand in sandwich complexes of first-row transi- 
tion metals with low oxidation state. The electrochemical properties such as the $\sigma$ - and $\pi$-delocalization as well as the $\pi$-accepting capability of a series of cobalt complexes containing two $\eta^{3}-N, N^{\prime}, N^{\prime \prime}$-chelating ligands have been discussed [9]. These sandwich complexes are generally formulated as $\left[\mathrm{M}\left(\mathrm{P}(2-\mathrm{py})_{3}\right)_{2}\right]^{2+}(\mathrm{M}=\mathrm{Mn}$, $\mathrm{Co}, \mathrm{Ni}, \mathrm{Cu}, \mathrm{Zn}, \mathrm{Ru}$ ) [8a-c]. However, only two examples of half-sandwich complexes have been reported, that is, $\left.\mathrm{ZnP}(2-\mathrm{py})_{3}\right)\left(\eta^{1}-\mathrm{NO}_{3}\right)\left(\eta^{2}-\mathrm{NO}_{3}\right) \quad[8 \mathrm{~d}] \quad$ and $\mathrm{RuCl}\left(\mathrm{PPh}_{3}\right) \mathrm{P}(2-\mathrm{py})_{3}[8 \mathrm{e}]$.

In this paper, we report our research on the synthesis, characterization, and reactivity of Group VI transition-metal half-sandwich complexes supported by $\eta^{3}-N, N^{\prime}, N^{\prime \prime}-\mathrm{P}(2-\text { py })_{3}$ ligation. Substitution of the neutral $\mathrm{CO}$ ligands in $\mathrm{P}(2-\mathrm{py})_{3} \mathrm{M}(\mathrm{CO})_{3}(\mathrm{M}=\mathrm{Cr}$ : 1a, Mo: 1b, W: 1c) by the isoelectronic $\mathrm{NO}^{+}$groups affords cationic species of the type $\left[\mathrm{P}(2-\mathrm{py})_{3} \mathrm{M}(\mathrm{CO})_{3-}\right.$ $\left.n(\mathrm{NO})_{n}\right]^{n+}(n=1,2)$. The dinitrosyl complexes, $[\mathrm{P}(2-$ py $\left.)_{3} \mathrm{M}(\mathrm{CO})(\mathrm{NO})_{2}\right]\left(\mathrm{BF}_{4}\right)_{2} \quad(\mathrm{M}=\mathrm{Mo}: 3 \mathbf{a}, \mathrm{W}: 3 \mathbf{b})$, have been proven to be strong Lewis acid precursors. In solution, complexes $\mathbf{3 a}$ and $\mathbf{3 b}$ release their $\mathrm{CO}$ and generate 16-electron species of the type $[\mathrm{P}(2-$ py) $\left.{ }_{3} \mathrm{M}(\mathrm{NO})_{2}\right]^{2+}$, which are capable of binding basic nitriles and aldehydes. Furthermore, $\mathbf{3 b}$ is also active in promoting the Diels-Alder reaction of cyclohexadiene and methyl vinyl ketone. The Diels-Alder reactions of 1,3-butadiene and methyl vinyl ketone [10] as well as the additions of silylated C-nucleophiles to carbonyl compounds [11] have also been observed to be catalyzed by the analogues Lewis acid precursor, $[\mathrm{HC}(2-$ py $\left.)_{3} \mathrm{~W}(\mathrm{CO})(\mathrm{NO})_{2}\right]\left(\mathrm{SbF}_{6}\right)_{2}$, where the $\mathrm{HC}(2-\mathrm{py})_{3}$ ligand is an $\eta^{3}-N, N^{\prime}, N^{\prime \prime}$ ligand.

Though catalysis using complexes containing pyridylphosphine ligands, $\mathrm{PPh}_{3-n}(2-\text { py })_{n}(n=1,2)$ has developed noticeably in the past two decades $[4,12]$, the application of $\mathrm{P}(2-\mathrm{py})_{3}$ in homogeneous catalysis is still limited [6]. We are therefore interested in exploring the catalytic aspects of $\mathrm{P}(2-\mathrm{py})_{3}$ in homogeneous systems.

\section{Experimental}

All manipulations were carried out either by use of standard Schlenk techniques or in a Vacuum Atmospheres glove-box unless specified otherwise. NMR spectra were recorded on a Varian Gemini-200 QNP or a Bruker AMX 400 QNP spectrometer, and chemical shifts were reported relatively to TMS for ${ }^{1} \mathrm{H}$ - and ${ }^{13} \mathrm{C}-\mathrm{NMR}$ spectra, $85 \% \mathrm{H}_{3} \mathrm{PO}_{4}$ for ${ }^{31} \mathrm{P}-\mathrm{NMR}$ spectra, and $\mathrm{CFCl}_{3}$ for ${ }^{19} \mathrm{~F}-\mathrm{NMR}$ spectra in $\mathrm{CDCl}_{3}, \mathrm{CD}_{3} \mathrm{NO}_{2}$, $\mathrm{CD}_{3} \mathrm{CN}$ and DMSO- $d_{6}$. Infrared spectra were obtained with a Perkin-Elmer 682 spectrometer. Elemental analyses were performed by the Microanalytical Laboratory of South Taiwan National Science Council Instrument Center. Mass spectra were obtained on a VG TRIO-2000 spectrometer.
All solvents were dried with use of standard procedures. Crotonaldehyde and methyl vinyl ketone were purified by vacuum-transferred from $\mathrm{CaH}_{2}$. Cyclohexadiene was used as purchased. All other materials were used as received without further purification. Tris(2pyridyl)phosphine was prepared by a modification of the reported procedure [6a]. $\mathrm{Cr}(\mathrm{CO})_{3}\left(\mathrm{CH}_{3} \mathrm{CN}\right)_{3}$ and $\mathrm{W}(\mathrm{CO})_{3}\left(\mathrm{CH}_{3} \mathrm{CN}\right)_{3}$ were prepared by following the literature methods [13].

\subsection{Synthesis of $\mathrm{P}(2-\mathrm{py})_{3} \mathrm{Cr}(\mathrm{CO})_{3}(\mathbf{1 a})$}

A suspension of $0.28 \mathrm{~g} \mathrm{Cr}(\mathrm{CO})_{6}(1.2 \mathrm{mmol})$ in $30 \mathrm{ml}$ of $\mathrm{CH}_{3} \mathrm{CN}$ was heated at reflux under $\mathrm{N}_{2}$ for $48 \mathrm{~h}$, tris-2-pyridylphosphine $(0.28 \mathrm{~g}, 1.1 \mathrm{mmol})$ was then added to the yellow solution. The reaction mixture was heated at reflux for another $12 \mathrm{~h}$. A purple-colored precipitate formed during reflux. After cooling, the resulting solution was concentrated, and the supernatant was decanted and the precipitate was washed with $\mathrm{CHCl}_{3}$ and dried in vacuo overnight to afford $\mathrm{P}(2$ py $)_{3} \mathrm{Cr}(\mathrm{CO})_{3}$. Yield $0.27 \mathrm{~g}\left(64 \%\right.$, based on $\mathrm{P}(2-\mathrm{py})_{3}$ added). ${ }^{1} \mathrm{H}-\mathrm{NMR}\left(\mathrm{CDCl}_{3}, 27^{\circ} \mathrm{C}, 200 \mathrm{MHz}\right): \delta 7.15(\mathrm{t})$, $7.62(\mathrm{t}), 8.05$ (t), 9.55 (d). ${ }^{31} \mathrm{P}-\mathrm{NMR}\left(\mathrm{CDCl}_{3}, 27^{\circ} \mathrm{C}, 80\right.$ $\mathrm{MHz}): \delta-12.4 \mathrm{ppm}$. IR $\left(\mathrm{CDCl}_{3}\right.$,): $v_{\mathrm{CO}}=1901$ (s), 1777 (br) $\mathrm{cm}^{-1}$. Anal. Calc. for $\mathrm{C}_{18} \mathrm{H}_{12} \mathrm{~N}_{3} \mathrm{O}_{3} \mathrm{PCr}$ : C, 53.29; H, 3.14; N, 10.21. Found: C, 53.88; H, 3.01; N, $10.47 \%$.

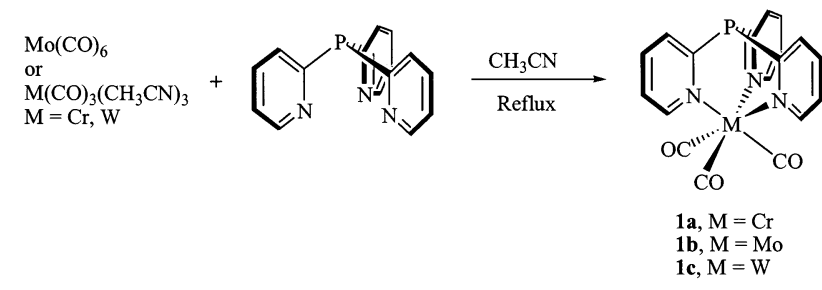

\subsection{Synthesis of $\mathrm{P}(2-p y)_{3} \mathrm{Mo}(\mathrm{CO})_{3}(\mathbf{1 b})$}

A mixture of Tris-2-pyridyl-phosphine (200 mg, 0.7 $\mathrm{mmol})$ and $\mathrm{Mo}(\mathrm{CO})_{6}(200 \mathrm{mg}, 0.7 \mathrm{mmol})$ in $10 \mathrm{ml}$ of $\mathrm{CH}_{3} \mathrm{CN}$ was heated at reflux under $\mathrm{N}_{2}$ for $8 \mathrm{~h}$ to afford a red precipitate. The crude product was filtered and washed with petroleum ether, and the solid was dried in vacuo overnight to give red $\mathrm{P}(2-\mathrm{py})_{3} \mathrm{Mo}(\mathrm{CO})_{3}$. Yield $288 \mathrm{mg}(89 \%) .{ }^{1} \mathrm{H}-\mathrm{NMR}\left(\mathrm{CDCl}_{3}, 27^{\circ} \mathrm{C}, 200 \mathrm{MHz}\right): \delta$ $7.20(\mathrm{t}), 7.70(\mathrm{t}), 8.08(\mathrm{t}), 9.45(\mathrm{~d}) .{ }^{31} \mathrm{P}-\mathrm{NMR}\left(\mathrm{CDCl}_{3}\right.$, $\left.27^{\circ} \mathrm{C}, 80 \mathrm{MHz}\right): \delta-3.2 \mathrm{ppm}$. IR $\left(\mathrm{CDCl}_{3}\right): v_{\mathrm{CO}}=1908$ (s), 1797 (br) $\mathrm{cm}^{-1}$. Anal. Calc. for $\mathrm{C}_{18} \mathrm{H}_{12} \mathrm{~N}_{3} \mathrm{O}_{3} \mathrm{PMo}$ : C, 48.35; H, 2.72; N, 9.41. Found: C, 48.56; H, 2.72; N, $9.44 \%$.

\subsection{Synthesis of $\mathrm{P}(2-\mathrm{py})_{3} W(\mathrm{CO})_{3}(\mathbf{1 c})$}

A suspension of $\mathrm{W}(\mathrm{CO})_{6}(220 \mathrm{mg}, 0.56 \mathrm{mmol})$ in 10 $\mathrm{ml}$ of $\mathrm{CH}_{3} \mathrm{CN}$ was heated at reflux under $\mathrm{N}_{2}$ for $24 \mathrm{~h}$, 
Tris-2-pyridylphosphine $(136 \mathrm{mg}, 0.51 \mathrm{mmol})$ was then added to the yellow solution. The reaction mixture was heated at reflux for another $12 \mathrm{~h}$. A maroon-colored precipitate formed during reflux. After cooling, the resulting solution was concentrated, and the supernatant was decanted and the precipitate was washed with petroleum ether and dried in vacuo overnight to afford $\mathrm{P}(2-\mathrm{py})_{3} \mathrm{~W}(\mathrm{CO})_{3}$. Yield $300 \mathrm{mg}\left(61 \%\right.$, based on $\mathrm{P}(2-\mathrm{py})_{3}$ added). ${ }^{1} \mathrm{H}-\mathrm{NMR}\left(\mathrm{CDCl}_{3}, 27^{\circ} \mathrm{C}, 200 \mathrm{MHz}\right): \delta 7.15(\mathrm{t})$, 7.75 (t), 8.15 (t), 9.45 (d). ${ }^{31} \mathrm{P}-\mathrm{NMR}\left(\mathrm{CDCl}_{3}, 27^{\circ} \mathrm{C}, 80\right.$ $\mathrm{MHz}): \delta-4.5 \mathrm{ppm}$. IR $\left(\mathrm{CDCl}_{3}\right): v_{\mathrm{CO}}=1899$ (s), 1784 (br) $\mathrm{cm}^{-1}$. Anal. Calc. for $\mathrm{C}_{18} \mathrm{H}_{12} \mathrm{~N}_{3} \mathrm{O}_{3} \mathrm{PW}$ : C, 40.49; $\mathrm{H}, 2.33$; N, 7.89. Found: C, 40.55; H, 2.27; N, 7.88\%.

\subsection{Synthesis of $\left[\mathrm{P}(2-\mathrm{py})_{3} \mathrm{Mo}(\mathrm{CO})_{2}(\mathrm{NO})\right]\left(\mathrm{BF}_{4}\right)(\mathbf{2 a})$}

To a suspension of $\mathrm{P}(2-\mathrm{py})_{3} \mathrm{Mo}(\mathrm{CO})_{3}(100 \mathrm{mg}, 0.225$ mmol) in $10 \mathrm{ml}$ of $\mathrm{CH}_{2} \mathrm{Cl}_{2}$ was added 1 equiv. of $\mathrm{NOBF}_{4}(26 \mathrm{mg}, 0.222 \mathrm{mmol})$. An immediate vigorous gas evolution was observed upon addition of $\mathrm{NOBF}_{4}$. The reaction mixture was allowed to stir at ambient temperature for $1 \mathrm{~h}$. The unreacted $\mathrm{P}(2-\mathrm{py})_{3} \mathrm{Mo}(\mathrm{CO})_{3}$ was filtered off from the dark red solution, and the solvent was removed from the filtrate to give an orange-red solid. The solid was then dried in vacuo overnight to give orange-red $\left[\mathrm{P}(2-\mathrm{py})_{3} \mathrm{Mo}(\mathrm{CO})_{2^{-}}\right.$ $(\mathrm{NO})]\left(\mathrm{BF}_{4}\right)$. Yield $95 \mathrm{mg}(79 \%)$. ${ }^{1} \mathrm{H}-\mathrm{NMR}\left(\mathrm{CD}_{3} \mathrm{CN}\right.$, $\left.27^{\circ} \mathrm{C}, 200 \mathrm{MHz}\right): \delta$ 7.55-7.65 (m), 8.06-8.18 (m), $8.28-8.41(m), 8.97(\mathrm{~d}, J=5.12 \mathrm{~Hz}), 9.20(\mathrm{~d}, J=4.76$ $\mathrm{Hz}) .{ }^{13} \mathrm{C}-\mathrm{NMR}\left(d_{6}\right.$-DMSO, $\left.27^{\circ} \mathrm{C}, 100 \mathrm{MHz}\right): \delta 222.0$ (CO). ${ }^{31} \mathrm{P}-\mathrm{NMR}\left(\mathrm{CD}_{3} \mathrm{CN}, 27^{\circ} \mathrm{C}, 80 \mathrm{MHz}\right): \delta-4.75$. ${ }^{19} \mathrm{~F}-\mathrm{NMR}\left(\mathrm{CD}_{3} \mathrm{CN}, 27^{\circ} \mathrm{C}, 376 \mathrm{MHz}\right): \delta-151.3 \mathrm{ppm}$. IR (KBr): $v_{\mathrm{CO}}=2046(\mathrm{~s}), 1940(\mathrm{br}) ; v_{\mathrm{NO}}=1668$ (s); $v_{\mathrm{BF}}=1060 \quad$ (br) $\mathrm{cm}^{-1}$. Anal. Calc. for $\mathrm{C}_{17} \mathrm{H}_{12} \mathrm{BF}_{4} \mathrm{~N}_{4} \mathrm{O}_{3}$ PMo: C, 38.23; H, 2.30; N, 10.41 . Found: C, 38.24; H, 2.26; N, 10.49\%.
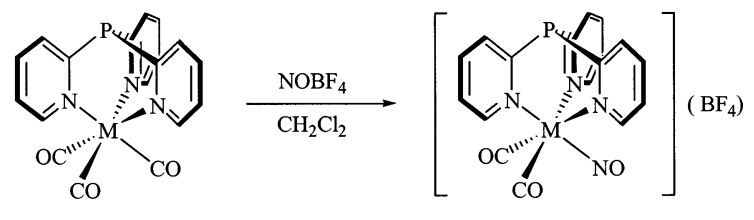

2a, $\mathrm{M}=\mathrm{Mo}$

2b, M $=\mathrm{W}$

\subsection{Synthesis of $\left[P(2-p y)_{3} W(C O)_{2}(N O)\right]\left(B F_{4}\right)(2 b)$}

To a suspension of $\mathrm{P}(2-\mathrm{py})_{3} \mathrm{~W}(\mathrm{CO})_{3}(100 \mathrm{mg}, 0.188$ mmol) in $10 \mathrm{ml}$ of $\mathrm{CH}_{2} \mathrm{Cl}_{2}$ was added 1 equiv. of $\mathrm{NOBF}_{4}(22 \mathrm{mg}, 0.188 \mathrm{mmol})$. An immediate vigorous gas evolution was observed upon addition of $\mathrm{NOBF}_{4}$. The reaction mixture was allowed to stir at ambient temperature for $1 \mathrm{~h}$. The suspended impurities were filtered off from the dark purple solution, and the solvent was removed from the filtrate to give a purple solid. The solid was then dried in vacuo overnight to give $\left[\mathrm{P}(2-\mathrm{py})_{3} \mathrm{~W}(\mathrm{CO})_{2}(\mathrm{NO})\right]\left(\mathrm{BF}_{4}\right)$. Yield $89 \mathrm{mg}(76 \%)$. ${ }^{1} \mathrm{H}-\mathrm{NMR}\left(\mathrm{CD}_{3} \mathrm{CN}, 27^{\circ} \mathrm{C}, 200 \mathrm{MHz}\right): \delta 7.55-7.65(\mathrm{~m})$, $8.02-8.20(\mathrm{~m}), 8.32-8.46(\mathrm{~m}), 9.05(\mathrm{~d}, J=5.12 \mathrm{~Hz})$, $9.28(\mathrm{~d}, J=5.12 \mathrm{~Hz}) .{ }^{13} \mathrm{C}-\mathrm{NMR}\left(d_{6}\right.$-DMSO, $27^{\circ} \mathrm{C}, 100$ $\mathrm{MHz}): \delta 222.1$ (CO). ${ }^{31} \mathrm{P}-\mathrm{NMR}\left(\mathrm{CD}_{3} \mathrm{CN}, 27^{\circ} \mathrm{C}, 80\right.$ $\mathrm{MHz}): \delta-8.07 .{ }^{19} \mathrm{~F}-\mathrm{NMR}\left(\mathrm{CD}_{3} \mathrm{CN}, 27^{\circ} \mathrm{C}, 376 \mathrm{MHz}\right)$ : $\delta-151.3$ ppm. IR (KBr): $v_{\mathrm{CO}}=2012$ (s), 1912 (br); $v_{\mathrm{NO}}=1650(\mathrm{~s}) ; v_{\mathrm{BF}}=1060$ (br) $\mathrm{cm}^{-1}$. Anal. Calc. for $\mathrm{C}_{17} \mathrm{H}_{12} \mathrm{BF}_{4} \mathrm{~N}_{4} \mathrm{O}_{3} \mathrm{PW}: \mathrm{C}, 38.81 ; \mathrm{H}, 1.91 ; \mathrm{N}, 9.01$. Found: C, 32.83; H, 1.94; N, 9.01\%.

\subsection{Synthesis of $\left[\mathrm{P}(2-\mathrm{py})_{3} \mathrm{Mo}(\mathrm{CO})(\mathrm{NO})_{2}\right]\left(\mathrm{BF}_{4}\right)_{2}(\mathbf{3 a})$}

To a suspension of $\left[\mathrm{P}(2-\mathrm{py})_{3} \mathrm{Mo}(\mathrm{CO})_{3}(100 \mathrm{mg}, 0.225\right.$ $\mathrm{mmol}$ ) in $5 \mathrm{ml}$ of $\mathrm{CH}_{3} \mathrm{NO}_{2}$ was added 2 equiv. of $\mathrm{NOBF}_{4}(53 \mathrm{mg}, 0.454 \mathrm{mmol})$. An immediate vigorous gas evolution was observed upon addition of $\mathrm{NOBF}_{4}$. The reaction mixture was allowed to stir at ambient temperature for $10 \mathrm{~min}$. The suspended impurities were filtered off from the dark green solution, and dried in vacuo overnight to give green solids of $[\mathrm{P}(2-$ py) $\left.{ }_{3} \mathrm{Mo}(\mathrm{CO})(\mathrm{NO})_{2}\right]\left(\mathrm{BF}_{4}\right)_{2}$. Yield $90 \mathrm{mg}(90 \%) .{ }^{1} \mathrm{H}-$ NMR $\left(\mathrm{CD}_{3} \mathrm{NO}_{2}, 27^{\circ} \mathrm{C}, 200 \mathrm{MHz}\right): \delta 7.87(\mathrm{~m}), 8.37(\mathrm{~m})$, $8.58(\mathrm{~m}), 9.08$ (d), 9.18 (d). ${ }^{31} \mathrm{P}-\mathrm{NMR}\left(\mathrm{CD}_{3} \mathrm{NO}_{2}, 27^{\circ} \mathrm{C}\right.$, $80 \mathrm{MHz}): \delta-5.8 \mathrm{ppm}$. IR $\left(\mathrm{CD}_{3} \mathrm{NO}_{2}\right): v_{\mathrm{CO}}=2037$ (br); $v_{\mathrm{NO}}=1839(\mathrm{~s}), 1738(\mathrm{~s}) ; v_{\mathrm{BF}}=1026$ (br) $\mathrm{cm}^{-1}$.
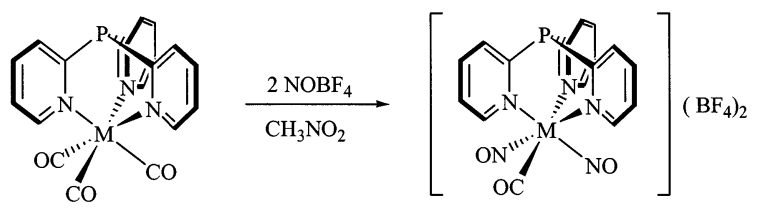

3a, $M=$ Mo $3 \mathbf{b}, \mathrm{M}=\mathrm{W}$

\subsection{Synthesis of $\left[P(2-p y)_{3} W(\mathrm{CO})(\mathrm{NO})_{2}\right]\left(\mathrm{BF}_{4}\right)_{2}(\mathbf{3 b})$}

To a suspension of $\mathrm{P}(2-\mathrm{py})_{3} \mathrm{~W}(\mathrm{CO})_{3}(100 \mathrm{mg}, 0.188$ mmol) in $5 \mathrm{ml}$ of $\mathrm{CH}_{3} \mathrm{NO}_{2}$ was added 2 equiv. of $\mathrm{NOBF}_{4}$ (44 mg, $0.376 \mathrm{mmol}$ ). An immediate vigorous gas evolution was observed upon addition of $\mathrm{NOBF}_{4}$. The reaction mixture was allowed to stir at ambient temperature for $30 \mathrm{~min}$. The suspended impurities were filtered off from the dark green solution. Recrystallizations of the crude product from $\mathrm{CH}_{3} \mathrm{NO}_{2}$-ether solution at $-18^{\circ} \mathrm{C}$ for $24 \mathrm{~h}$ gave green crystals of $\left[\mathrm{P}(2-\mathrm{py})_{3} \mathrm{~W}(\mathrm{CO})(\mathrm{NO})_{2}\right]\left(\mathrm{BF}_{4}\right)_{2}$. Yield $75 \mathrm{mg}(56 \%) .{ }^{1} \mathrm{H}-$ NMR $\left(\mathrm{CD}_{3} \mathrm{NO}_{2}, 27^{\circ} \mathrm{C}, 200 \mathrm{MHz}\right): \delta 7.89-7.96(\mathrm{~m})$, 8.43-8.50 (m), 8.64-8.74 (m), 9.25-9.43 (m). ${ }^{13} \mathrm{C}-$ NMR $\left(\mathrm{CD}_{3} \mathrm{NO}_{2}, 27^{\circ} \mathrm{C}, 50 \mathrm{MHz}\right): \delta 192.2(\mathrm{CO}) .{ }^{31} \mathrm{P}-$ NMR $\left(\mathrm{CD}_{3} \mathrm{NO}_{2}, 27^{\circ} \mathrm{C}, 80 \mathrm{MHz}\right): \delta-10.58 .{ }^{19} \mathrm{~F}-\mathrm{NMR}$ $\left(\mathrm{CD}_{3} \mathrm{NO}_{2}, 27^{\circ} \mathrm{C}, 188 \mathrm{MHz}\right): \delta-151.7 \mathrm{ppm}$. IR (KBr): $v_{\mathrm{CO}}=2148(\mathrm{br}) ; v_{\mathrm{NO}}=1844(\mathrm{~s}), 1758(\mathrm{~s}) ; v_{\mathrm{BF}}=1030$ (br) $\mathrm{cm}^{-1}$. Anal. Calc. for $\mathrm{C}_{16} \mathrm{H}_{12} \mathrm{~B}_{2} \mathrm{~F}_{8} \mathrm{~N}_{5} \mathrm{O}_{3} \mathrm{PW}$ : C, 26.74; H, 1.78; N, 9.70. Found: C, 27.04; H, 1.70; N, $9.85 \%$. 
2.8. Synthesis of $\left[\mathrm{P}(2-\mathrm{py})_{3} \mathrm{M}(\mathrm{L})(\mathrm{NO})_{2}\right]\left(\mathrm{BF}_{4}\right)_{2}$, where $\mathrm{M}=\mathrm{Mo}$ and $\mathrm{W} ; \mathrm{L}=\mathrm{CH}_{3} \mathrm{CN}$ and $\mathrm{CH}_{3} \mathrm{CH}_{2} \mathrm{CN}$

A typical procedure for the synthesis of $[\mathrm{P}(2-$ py $\left.)_{3} \mathrm{M}(\mathrm{L})(\mathrm{NO})_{2}\right]\left(\mathrm{BF}_{4}\right)_{2}$ is described as follows. To a suspension of $\mathrm{P}(2-\mathrm{py})_{3} \mathrm{Mo}(\mathrm{CO})_{3}(50 \mathrm{mg}, 0.112 \mathrm{mmol})$ in $5 \mathrm{ml}$ of $\mathrm{CH}_{3} \mathrm{CN}$ was added 2 equiv. of $\mathrm{NOBF}_{4}(26 \mathrm{mg}$, $0.222 \mathrm{mmol})$. An immediate vigorous gas evolution was observed upon addition of $\mathrm{NOBF}_{4}$. The reaction mixture was allowed to stir at ambient temperature for 30 min. The suspended impurities were filtered off from the dark green solution, and $5 \mathrm{ml}$ of dry diethyl ether ( 5 $\mathrm{ml}$ ) was added to the filtrate. The resulting solution was allowed to sit at $-18^{\circ} \mathrm{C}$ for $24 \mathrm{~h}$ to give green crystalline solids of $\left[\mathrm{P}(2-\mathrm{py})_{3} \mathrm{Mo}\left(\mathrm{CH}_{3} \mathrm{CN}\right)(\mathrm{NO})_{2}\right]\left(\mathrm{BF}_{4}\right)_{2}$. Spectroscopic data are given below.

\subsection{1. $\left[\mathrm{P}(2-\mathrm{py})_{3} \mathrm{Mo}\left(\mathrm{CH}_{3} \mathrm{CN}\right)(\mathrm{NO})_{2}\right]\left(\mathrm{BF}_{4}\right)_{2}(\mathbf{4 a})$}

${ }^{1} \mathrm{H}-\mathrm{NMR}\left(\mathrm{CD}_{3} \mathrm{NO}_{2}, 27^{\circ} \mathrm{C}, 200 \mathrm{MHz}\right): \delta 7.81-7.91$ (m), 8.30-8.40 (m), 8.56-8.72 (m), $9.08(\mathrm{~d}, J=5.12$ $\mathrm{Hz}), 9.28(\mathrm{~d}, J=5.20 \mathrm{~Hz}), 2.62\left(\mathrm{~s}, \mathrm{CH}_{3} \mathrm{CN}\right) .{ }^{13} \mathrm{C}-\mathrm{NMR}$ $\left(\mathrm{CD}_{3} \mathrm{NO}_{2}, 27^{\circ} \mathrm{C}, 50 \mathrm{MHz}\right): \delta 136.5\left(\mathrm{CH}_{3} \mathrm{CN}\right), 4.3$ $\left(\mathrm{CH}_{3} \mathrm{CN}\right) .{ }^{31} \mathrm{P}-\mathrm{NMR}\left(\mathrm{CD}_{3} \mathrm{NO}_{2}, 27^{\circ} \mathrm{C}, 80 \mathrm{MHz}\right): \delta-$ 6.26. ${ }^{19} \mathrm{~F}-\mathrm{NMR}\left(\mathrm{CD}_{3} \mathrm{NO}_{2}, 27^{\circ} \mathrm{C}, 188 \mathrm{MHz}\right): \delta-151.5$ ppm. IR (KBr): $v_{\mathrm{CN}}=2294$ (br); $v_{\mathrm{NO}}=1834$ (s), 1732 (s) $\mathrm{cm}^{-1}$. Anal. Calc. for $\mathrm{C}_{17} \mathrm{H}_{15} \mathrm{~B}_{2} \mathrm{~F}_{8} \mathrm{~N}_{6} \mathrm{O}_{2}$ PMo: C, $28.20 ; \mathrm{H}, 2.10 ; \mathrm{N}, 11.52$. Found: C, 28.21; H, 2.09; N, $11.61 \%$.
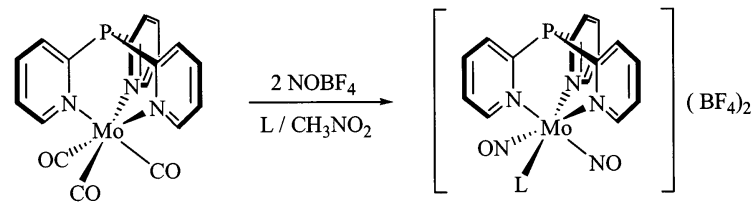

4a, $\mathrm{M}=\mathrm{Mo} ; \mathrm{L}=\mathrm{CH}_{3} \mathrm{CN}$ 4b, $\mathrm{M}=\mathrm{Mo} ; \mathrm{L}=\mathrm{C}_{2} \mathrm{H}_{5} \mathrm{CN}$ 4c, $\mathrm{M}=\mathrm{Mo} ; \mathrm{L}=\mathrm{CH}_{3} \mathrm{CH}=\mathrm{CHCH}=\mathrm{O}$ 5a, $\mathrm{M}=\mathrm{W} ; \mathrm{L}=\mathrm{CH}_{3} \mathrm{CN}$

5b, $\mathrm{M}=\mathrm{W} ; \mathrm{L}=\mathrm{C}_{2} \mathrm{H}_{5} \mathrm{CN}$ 5c, $\mathrm{M}=\mathrm{W} ; \mathrm{L}=\mathrm{CH}_{3} \mathrm{CH}=\mathrm{CHCH}=\mathrm{O}$ 5d, $\mathrm{M}=\mathrm{W} ; \mathrm{L}=\left(\mathrm{C}_{6} \mathrm{H}_{5}\right) \mathrm{CH}=\mathrm{O}$

\subsection{2. $\left[\mathrm{P}(2-\mathrm{py})_{3} \mathrm{Mo}\left(\mathrm{CH}_{3} \mathrm{CH}_{2} \mathrm{CN}\right)(\mathrm{NO})_{2}\right]\left(\mathrm{BF}_{4}\right)_{2}(\mathbf{4 b})$}

${ }^{1} \mathrm{H}-\mathrm{NMR}\left(\mathrm{CD}_{3} \mathrm{NO}_{2}, 27^{\circ} \mathrm{C}, 200 \mathrm{MHz}\right): \delta 7.81-7.92$ (m), 8.29-8.41 (m), 8.52-8.67 (m), $9.08(\mathrm{~d}, J=5.22$ $\mathrm{Hz}), 9.17$ (d, $J=5.20 \mathrm{~Hz}), 2.98$ (q, $J=7.40 \mathrm{~Hz}$, $\mathrm{CH}_{3} \mathrm{CH}_{2} \mathrm{CN}$ ), 1.36 (t, $\left.\mathrm{J}=7.62 \mathrm{~Hz}, \mathrm{CH}_{3} \mathrm{CH}_{2} \mathrm{CN}\right) .{ }^{13} \mathrm{C}-$ NMR $\quad\left(\mathrm{CD}_{3} \mathrm{NO}_{2}, \quad 27^{\circ} \mathrm{C}, \quad 50 \quad \mathrm{MHz}\right): \quad \delta \quad 139.5$ $\left(\mathrm{CH}_{3} \mathrm{CH}_{2} \mathrm{CN}\right), 13.8\left(\mathrm{CH}_{3} \mathrm{CH}_{2} \mathrm{CN}\right), 9.4\left(\mathrm{CH}_{3} \mathrm{CH}_{2} \mathrm{CN}\right)$. ${ }^{31} \mathrm{P}-\mathrm{NMR}\left(\mathrm{CD}_{3} \mathrm{NO}_{2}, 2{ }^{\circ} \mathrm{C}, 80 \mathrm{MHz}\right): \delta-5.91 .{ }^{19} \mathrm{~F}-$ NMR $\left(\mathrm{CD}_{3} \mathrm{NO}_{2}, 27^{\circ} \mathrm{C}, 188 \mathrm{MHz}\right): \delta-151.7 \mathrm{ppm}$. IR $(\mathrm{KBr}): v_{\mathrm{CN}}=2294(\mathrm{br}) ; v_{\mathrm{NO}}=1830(\mathrm{~s}), 1728(\mathrm{~s}) \mathrm{cm}^{-1}$. Anal. Calc. for $\mathrm{C}_{19} \mathrm{H}_{20} \mathrm{~B}_{2} \mathrm{~F}_{8} \mathrm{~N}_{7} \mathrm{O}_{4} \mathrm{PMo}$ : C, 31.93; H, 2.83; N, 13.57. Found: C, $32.07 \mathrm{H}, 2.81$; N, 13.78\%.

\subsection{3. $\left[\mathrm{P}(2-p y)_{3} \mathrm{~W}\left(\mathrm{CH}_{3} \mathrm{CN}\right)(\mathrm{NO})_{2}\right]\left(\mathrm{BF}_{4}\right)_{2}(\mathbf{5 a})$}

${ }^{1} \mathrm{H}-\mathrm{NMR}\left(\mathrm{CD}_{3} \mathrm{NO}_{2}, 27^{\circ} \mathrm{C}, 200 \mathrm{MHz}\right): \delta 7.82-7.95$ (m), 8.33-8.45 (m), 8.58-8.71 (m), 9.17-9.22 (m), 2.66 (s, $\left.\mathrm{CH}_{3} \mathrm{CN}\right) \cdot{ }^{13} \mathrm{C}-\mathrm{NMR}\left(\mathrm{CD}_{3} \mathrm{NO}_{2}, 27^{\circ} \mathrm{C}, 50 \mathrm{MHz}\right): \delta$ $138.1\left(\mathrm{CH}_{3} \mathrm{CN}\right), 4.8\left(\mathrm{CH}_{3} \mathrm{CN}\right) .{ }^{31} \mathrm{P}-\mathrm{NMR}\left(\mathrm{CD}_{3} \mathrm{NO}_{2}\right.$, $\left.27^{\circ} \mathrm{C}, 80 \mathrm{MHz}\right): \delta-10.75 .{ }^{19} \mathrm{~F}-\mathrm{NMR}\left(\mathrm{CD}_{3} \mathrm{NO}_{2}, 27^{\circ} \mathrm{C}\right.$, $188 \mathrm{MHz}): \delta-151.3 \mathrm{ppm}$. IR (KBr): $v_{\mathrm{CN}}=2296$ (br); $v_{\mathrm{NO}}=1802(\mathrm{~s}), 1716$ (s) $\mathrm{cm}^{-1}$. Anal. Calc. for $\mathrm{C}_{17} \mathrm{H}_{15} \mathrm{~B}_{2} \mathrm{~F}_{8} \mathrm{~N}_{6} \mathrm{O}_{2} \mathrm{PW}$ : C, 28.20; H, 2.10; N, 11.52 . Found: C, 28.21; H, 2.09; N, 11.61\%.

\subsection{4. $\left[\mathrm{P}(2-\mathrm{py})_{3} \mathrm{~W}\left(\mathrm{CH}_{3} \mathrm{CH}_{2} \mathrm{CN}\right)(\mathrm{NO})_{2}\right]\left(\mathrm{BF}_{4}\right)_{2}(\mathbf{5 b})$}

${ }^{1} \mathrm{H}-\mathrm{NMR}\left(\mathrm{CD}_{3} \mathrm{NO}_{2}, 27^{\circ} \mathrm{C}, 200 \mathrm{MHz}\right): \delta 7.82-7.96$ (m), 8.34-8.46 (m), 8.52-8.72 (m), 9.16-9.22 (m), 3.00 (q, $J=7.52 \mathrm{~Hz}, \mathrm{CH}_{3} \mathrm{CH}_{2} \mathrm{CN}$ ), 1.37 (t, $J=7.50 \mathrm{~Hz}$, $\left.\mathrm{CH}_{3} \mathrm{CH}_{2} \mathrm{CN}\right) .{ }^{13} \mathrm{C}-\mathrm{NMR}\left(\mathrm{CD}_{3} \mathrm{NO}_{2}, 27^{\circ} \mathrm{C}, 50 \mathrm{MHz}\right): \delta$ $141.0 \quad\left(\mathrm{CH}_{3} \mathrm{CH}_{2} \mathrm{CN}\right), \quad 14.1 \quad\left(\mathrm{CH}_{3} \mathrm{CH}_{2} \mathrm{CN}\right), \quad 9.2$ $\left(\mathrm{CH}_{3} \mathrm{CH}_{2} \mathrm{CN}\right) .{ }^{31} \mathrm{P}-\mathrm{NMR}\left(\mathrm{CD}_{3} \mathrm{NO}_{2}, 27^{\circ} \mathrm{C}, 80 \mathrm{MHz}\right): \delta$ $-10.71 .{ }^{19} \mathrm{~F}-\mathrm{NMR}\left(\mathrm{CD}_{3} \mathrm{NO}_{2}, 27^{\circ} \mathrm{C}, 188 \mathrm{MHz}\right): \delta-$ $151.3 \mathrm{ppm}$. IR (KBr): $v_{\mathrm{CN}}=2284(\mathrm{br}) ; v_{\mathrm{NO}}=1790(\mathrm{~s})$, 1688 (s) $\mathrm{cm}^{-1}$. Anal. Calc. for $\mathrm{C}_{18} \mathrm{H}_{17} \mathrm{~B}_{2} \mathrm{~F}_{8} \mathrm{~N}_{6} \mathrm{O}_{2} \mathrm{PW}$ : C, 29.37; H, 2.29; N, 11.16. Found: C, 29.30; H, 2.32; N, $11.39 \%$.

2.9. Reactions of $\left[\mathrm{P}(2-\mathrm{py})_{3} \mathrm{M}(\mathrm{CO})(\mathrm{NO})_{2}\right]\left(\mathrm{BF}_{4}\right)_{2}$ with aldehydes, where $M=M o$ and $W$;

aldehyde $=\mathrm{CH}_{3} \mathrm{CH}=\mathrm{CHCHO}$ and $\mathrm{C}_{6} \mathrm{H}_{5} \mathrm{CHO}$

A typical procedure for the synthesis of $[\mathrm{P}(2-$ py) $\left.{ }_{3} \mathrm{M}(\mathrm{L})(\mathrm{NO})_{2}\right]\left(\mathrm{BF}_{4}\right)_{2}$ is described as follows. A solution of $\mathrm{P}(2-\mathrm{py})_{3} \mathrm{Mo}(\mathrm{CO})_{3}(50 \mathrm{mg}, 0.112 \mathrm{mmol})$ and $\mathrm{NOBF}_{4}(9 \mathrm{mg}, 0.084 \mathrm{mmol})$ in $0.5 \mathrm{ml}$ of $\mathrm{CD}_{3} \mathrm{NO}_{2}$ was allowed to react for $20 \mathrm{~min}$ in a $5 \mathrm{~mm}$ Wilmad $528 \mathrm{pp}$ NMR tube equipped with an air-free J. Young Valve. A slight excess of crotonaldehyde $(50 \mu \mathrm{l})$ was then added into the reaction tube. The solution was allowed to react at room temperature for one day until no starting metal material resonances were observed by the ${ }^{1} \mathrm{H}-\mathrm{NMR}$. Spectroscopic data is given below.

\subsection{1. $\left[\mathrm{P}(2-\mathrm{py})_{3} \mathrm{Mo}\left(\mathrm{CH}_{3} \mathrm{CH}=\mathrm{CHCHO}\right)(\mathrm{NO})_{2}\right]\left(\mathrm{BF}_{4}\right)_{2}$ (4c)}

${ }^{1} \mathrm{H}-\mathrm{NMR}\left(\mathrm{CD}_{3} \mathrm{NO}_{2}, 27^{\circ} \mathrm{C}, 200 \mathrm{MHz}\right): \delta 7.81-7.95$ (m), 8.30-8.45 (m), 8.54-8.69 (m), 8.90-9.05 (m), 9.91 (d, $\left.J=8.70 \mathrm{~Hz}, \mathrm{CH}_{3} \mathrm{CH}=\mathrm{CHCHO}\right), 7.98-8.15$ (m, $\left.\mathrm{CH}_{3} \mathrm{CH}=\mathrm{CHCHO}\right), \quad 6.64-6.76 \quad\left(\mathrm{~m}, \quad \mathrm{CH}_{3} \mathrm{CH}=\right.$ $\mathrm{CHCHO}), 2.34\left(\mathrm{~d}, J=6.50 \mathrm{~Hz}, \mathrm{CH}_{3} \mathrm{CH}=\mathrm{CHCHO}\right)$. ${ }^{13} \mathrm{C}-\mathrm{NMR} \quad\left(\mathrm{CD}_{3} \mathrm{NO}_{2}, \quad 27^{\circ} \mathrm{C}, \quad 50 \quad \mathrm{MHz}\right): \quad \delta \quad 211.5$ $\left(\mathrm{CH}_{3} \mathrm{CH}=\mathrm{CHCHO}\right), 177.6\left(\mathrm{CH}_{3} \mathrm{CH}=\mathrm{CHCHO}\right), 134.4$ $\left(\mathrm{CH}_{3} \mathrm{CH}=\mathrm{CHCHO}\right), 21.7 \mathrm{ppm}\left(\mathrm{CH}_{3} \mathrm{CH}=\mathrm{CHCHO}\right)$.

\subsection{2. $\left[\mathrm{P}(2-\mathrm{py})_{3} \mathrm{~W}\left(\mathrm{CH}_{3} \mathrm{CH}=\mathrm{CHCHO}\right)(\mathrm{NO})_{2}\right]\left(\mathrm{BF}_{4}\right)_{2} \quad(\mathbf{5 c})$}

${ }^{1} \mathrm{H}-\mathrm{NMR}\left(\mathrm{CD}_{3} \mathrm{NO}_{2} 27^{\circ} \mathrm{C}, 200 \mathrm{MHz}\right): \delta 7.75-8.00$ (m), 8.30-8.45 (m), 8.55-8.72 (m), 8.87 (d, $J=5.80$ $\mathrm{Hz}), 9.10(\mathrm{~d}, J=5.76 \mathrm{~Hz}), 9.16-9.22(\mathrm{~m}), 9.96(\mathrm{~d}$, $\left.J=8.90 \quad \mathrm{~Hz}, \quad \mathrm{CH}_{3} \mathrm{CH}=\mathrm{CHCHO}\right), \quad 8.11-8.29 \quad(\mathrm{~m}$, $\left.\mathrm{CH}_{3} \mathrm{CH}=\mathrm{CHCHO}\right), 6.70-6.82\left(\mathrm{~m}, \mathrm{CH}_{3} \mathrm{CH}=\mathrm{CHCHO}\right)$, $2.37\left(\mathrm{~d}, J=6.88 \mathrm{~Hz}, \mathrm{CH}_{3} \mathrm{CH}=\mathrm{CHCHO}\right) .{ }^{13} \mathrm{C}-\mathrm{NMR}$ 
$\begin{array}{lllllll}\left(\mathrm{CD}_{3} \mathrm{NO}_{2},\right. & 27 & { }^{\circ} \mathrm{C}, & 50 & \mathrm{MHz}): & \delta & 211.1\end{array}$ $\left(\mathrm{CH}_{3} \mathrm{CH}=\mathrm{CHCHO}\right), 180.7\left(\mathrm{CH}_{3} \mathrm{CH}=\mathrm{CHCHO}\right), 134.8$ $\left(\mathrm{CH}_{3} \mathrm{CH}=\mathrm{CHCHO}\right), 22.2 \mathrm{ppm}\left(\mathrm{CH}_{3} \mathrm{CH}=\mathrm{CHCHO}\right)$.

2.9.3. $\left[\mathrm{P}(2-\mathrm{py})_{3} \mathrm{~W}\left(\mathrm{C}_{6} \mathrm{H}_{5} \mathrm{CHO}\right)(\mathrm{NO})_{2}\right]\left(\mathrm{BF}_{4}\right)_{2}(\mathbf{5 d})$

${ }^{1} \mathrm{H}-\mathrm{NMR}\left(\mathrm{CD}_{3} \mathrm{NO}_{2}, 27^{\circ} \mathrm{C}, 200 \mathrm{MHz}\right): \delta 10.60(\mathrm{~s}$, $\mathrm{C}_{6} \mathrm{H}_{5} \mathrm{CHO}$ ), 8.00-8.20 ppm (m, $\mathrm{C}_{6} H_{5} \mathrm{CHO}$ ).

\subsection{Catalytic Diels-Alder reactions of cyclohexadiene and methyl vinyl ketone}

All Diels-Alder reactions were carried out in the same manner. In a typical procedure, $10 \mathrm{mg}(14.0$ $\mu \mathrm{mol})$ of $\left[\mathrm{P}(2-\mathrm{py})_{3} \mathrm{~W}(\mathrm{CO})(\mathrm{NO})_{2}\right]\left(\mathrm{BF}_{4}\right)_{2}(3 \mathbf{b})$ in $10 \mathrm{ml}$ of $\mathrm{CH}_{2} \mathrm{Cl}_{2}$ was placed in a $25-\mathrm{ml}$ flask. The flask was cooled to $0^{\circ} \mathrm{C}$ before the addition of $0.4 \mathrm{ml}$ of methyl vinyl ketone $(4.8 \mathrm{mmol})$. Cyclohexadiene $(0.53 \mathrm{ml}, 5.6$ $\mathrm{mmol}$ ) was then added dropwise to the reaction mixture over 2-3 min. After $24 \mathrm{~h}$, the solution was subjected to a rotary evaporator to remove all volatiles. The crude products were then purified by column chromatography using 20:1 hexane-ethylacetate as eluent. Yield $0.61 \mathrm{~g}$ $(85 \%)$. All Diels-Alder products were identified by NMR comparison to authentic material made as described in the literature. The percentage conversion and isomer ratios were determined by ${ }^{1} \mathrm{H}-\mathrm{NMR}$ integration of the enone aldehydic proton and methyl protons of the cyclohexadiene adducts.

\subsection{1. $X$-ray crystallographic analyses of $\mathrm{P}(2-p y)_{3} \mathrm{Mo}(\mathrm{CO})_{3} \cdot \mathrm{CH}_{2} \mathrm{Cl}_{2}$ and $\left[\mathrm{P}(2-\mathrm{py})_{3} \mathrm{Mo}\left(\mathrm{CH}_{3} \mathrm{CH}_{2} \mathrm{CN}\right)(\mathrm{NO})_{2}\right]\left(\mathrm{BF}_{4}\right)_{2} \cdot \mathrm{CH}_{3} \mathrm{NO}_{2}$}

General operation procedures have been reviewed. Some selected crystallographic data and data collection parameters are summarized in Tables 1 and 2. Suitable single crystals of $\mathrm{P}(2-\mathrm{py})_{3} \mathrm{Mo}(\mathrm{CO})_{3} \cdot \mathrm{CH}_{2} \mathrm{Cl}_{2}$ and $[\mathrm{P}(2-$ py) $\left.{ }_{3} \mathrm{Mo}\left(\mathrm{CH}_{3} \mathrm{CH}_{2} \mathrm{CN}\right)(\mathrm{NO})_{2}\right]\left(\mathrm{BF}_{4}\right)_{2} \cdot \mathrm{CH}_{3} \mathrm{NO}_{2}$ were grown at $-18^{\circ} \mathrm{C}$ from $\mathrm{CH}_{2} \mathrm{Cl}_{2}$ and $\mathrm{CH}_{3} \mathrm{CH}_{2} \mathrm{CN}-$ $\mathrm{CH}_{3} \mathrm{NO}_{2}$ solutions, respectively, for X-ray single-crystal structure determination. A crystal of $\mathrm{P}(2$ py) ${ }_{3} \mathrm{Mo}(\mathrm{CO})_{3} \cdot \mathrm{CH}_{2} \mathrm{Cl}_{2}$ with dimensions of $0.40 \times 0.50 \times$ $0.55 \mathrm{~mm}^{3}$ and a crystal of $\left[\mathrm{P}(2-\mathrm{py})_{3} \mathrm{Mo}\left(\mathrm{CH}_{3} \mathrm{CH}_{2} \mathrm{CN}\right)-\right.$ $\left.(\mathrm{NO})_{2}\right]\left(\mathrm{BF}_{4}\right)_{2} \cdot \mathrm{CH}_{3} \mathrm{NO}_{2}$ with dimensions of $0.15 \times$ $0.25 \times 0.35 \mathrm{~mm}^{3}$ were mounted in capillary tubes under a nitrogen atmosphere. Data were recorded at ambient temperature on an Enraf-Nonius CAD4 diffractometer using graphite-monochromatized $\mathrm{Mo}-\mathrm{K}_{\alpha}$ radiation. Unit-cell dimensions were determined from 25 well-centered reflections $\left(20.84 \leq 2 \theta \leq 38.10^{\circ}\right)$ for $\mathrm{P}(2$ py) $)_{3} \mathrm{Mo}(\mathrm{CO})_{3} \cdot \mathrm{CH}_{2} \mathrm{Cl}_{2}$, and from 25 well-centered reflections $\left(14.74 \leq 2 \theta \leq 29.36^{\circ}\right)$ for $\left[\mathrm{P}(2-p y)_{3} \mathrm{Mo}-\right.$ $\left.\left(\mathrm{CH}_{3} \mathrm{CH}_{2} \mathrm{CN}\right)(\mathrm{NO})_{2}\right]\left(\mathrm{BF}_{4}\right)_{2} \cdot \mathrm{CH}_{3} \mathrm{NO}_{2}$. Three intensity standards were measured for every $1 \mathrm{~h}$ of exposure time and declined systematically by $2 \%$ during the course of the measurements. Both structures were solved using the Patterson heavy-atom method [14] and refined by a full-matrix least-square procedure using NRCVAX [15] to reveal the positions of molybdenum atoms. Systematic absences and subsequent least-squares refinement indicated a monoclinic $C 2 / c$ space group for $\mathrm{P}(2-$ py) ${ }_{3} \mathrm{Mo}(\mathrm{CO})_{3} \cdot \mathrm{CH}_{2} \mathrm{Cl}_{2}$ and a monoclinic $P 2_{1} / c$ space group for $\left[\mathrm{P}(2-\mathrm{py})_{3} \mathrm{Mo}\left(\mathrm{CH}_{3} \mathrm{CH}_{2} \mathrm{CN}\right)(\mathrm{NO})_{2}\right]\left(\mathrm{BF}_{4}\right)_{2}$. $\mathrm{CH}_{3} \mathrm{NO}_{2}$. The remaining atoms were located in suc-

Table 1

Crystallographic data for $\mathrm{P}(2-\mathrm{py})_{3} \mathrm{Mo}(\mathrm{CO})_{3} \cdot \mathrm{CH}_{2} \mathrm{Cl}_{2}$ and $[\mathrm{P}(2-$ py) $\left.{ }_{3} \mathrm{Mo}\left(\mathrm{CH}_{3} \mathrm{CH}_{2} \mathrm{CN}\right)(\mathrm{NO})_{2}\right]\left(\mathrm{BF}_{4}\right)_{2} \cdot \mathrm{CH}_{3} \mathrm{NO}_{2}$

\begin{tabular}{|c|c|c|}
\hline Formula & $\mathrm{C}_{19} \mathrm{H}_{14} \mathrm{~N}_{3} \mathrm{O}_{3} \mathrm{MoPCl}_{2}$ & $\mathrm{C}_{19} \mathrm{H}_{20} \mathrm{~N}_{7} \mathrm{O}_{4} \mathrm{MoB}_{2} \mathrm{~F}_{8}$ \\
\hline FW & 530.15 & 710.93 \\
\hline Diffractometer & Nonius & Nonius \\
\hline Space group & Monoclinic, $C 2 / c$ & Monoclinic, $P 2_{1 / c}$ \\
\hline$a(\AA)$ & $26.304(6)$ & $17.754(4)$ \\
\hline$b(\AA)$ & $10.8570(20)$ & $11.204(3)$ \\
\hline$c(\AA)$ & $17.087(6)$ & $16.025(3)$ \\
\hline$\beta\left(^{\circ}\right)$ & $121.53(3)$ & $115.292(18)$ \\
\hline$V\left(\AA^{3}\right)$ & $4159.6(19)$ & $2882.2(12)$ \\
\hline$Z$ & 8 & 4 \\
\hline$D_{\text {calc. }}\left(\mathrm{g} \mathrm{cm}^{-3}\right)$ & 1.693 & 1.638 \\
\hline$\lambda(\AA)$ & 0.7107 & 0.7107 \\
\hline$F(000)$ & 2101 & 1410 \\
\hline $\begin{array}{l}\text { Unit cell detn: \# } \\
(2 \theta \text { range })\end{array}$ & $25\left(20.84-38.10^{\circ}\right)$ & $25\left(14.74-29.36^{\circ}\right)$ \\
\hline Scan type & $\theta / 2 \theta$ & $\theta / 2 \theta$ \\
\hline Scan width $\left(^{\circ}\right)$ & $\begin{array}{l}2(0.65+ \\
0.35 \tan \theta)\end{array}$ & $\begin{array}{l}2(0.60+ \\
0.35 \tan \theta)\end{array}$ \\
\hline Scan speed $\left({ }^{\circ} \min ^{-1}\right)$ & $2.06-8.24$ & $2.06-8.24$ \\
\hline $2 \theta(\max )$ & 50.0 & 50.0 \\
\hline$h, k, l$ ranges & $\begin{array}{l}(-31 ; 26) \\
(0 ; 12),(0 ; 20)\end{array}$ & $\begin{array}{l}((21 ; 19),(0 ; 13), \\
(0 ; 18)\end{array}$ \\
\hline$\mu\left(\mathrm{cm}^{-1}\right)$ & 6.319 & 5.713 \\
\hline Crystal size (mm) & $0.40 \times 0.50 \times 0.55$ & $0.15 \times 0.25 \times 0.35$ \\
\hline Transmission & $0.881 ; 1.000$ & $0.965 ; 1.000$ \\
\hline Temperature (K) & 298 & 298 \\
\hline $\begin{array}{l}\text { Number of meas } \\
\text { reflections }\end{array}$ & 3730 & 5064 \\
\hline $\begin{array}{l}\text { Number of obsed } \\
\text { reflections }(I>2.0 \sigma(I))\end{array}$ & 3105 & 2326 \\
\hline $\begin{array}{l}\text { Number of unique } \\
\text { reflections a }\end{array}$ & 3645 & 5064 \\
\hline$R_{f} ; R_{w}(I>2.0 \sigma(I))^{\mathrm{b}}$ & $0.029 ; 0.030$ & $0.064 ; 0.063$ \\
\hline$R_{f} ; R_{w}$ (all reflections) & $0.037 ; 0.036$ & $0.147 ; 0.067$ \\
\hline Goodness of-fit ${ }^{c}$ & 1.47 & 2.88 \\
\hline Refinement program & NRCVAX & NRCVAX \\
\hline Number of atoms & 47 & 62 \\
\hline $\begin{array}{l}\text { Number of refined } \\
\text { parameters }\end{array}$ & $\begin{array}{l}272(3105 \text { out of } \\
3645)\end{array}$ & $\begin{array}{l}380(2326 \text { out of } \\
5064)\end{array}$ \\
\hline Minimize function & $\Sigma\left(w\left|F_{\mathrm{o}}-F_{\mathrm{c}}\right|^{2}\right)$ & $\Sigma\left(w\left|F_{\mathrm{o}}-F_{\mathrm{c}}\right|^{2}\right)$ \\
\hline$(\Delta / \sigma) \max$ & 0.0117 & 0.0372 \\
\hline $\begin{array}{l}\text { Residual in final } D \text {-map } \\
\max \min \left(\mathrm{e} \AA^{3}\right)\end{array}$ & $-0.730 ; 0.770$ & $(0.690 ; 0.810$ \\
\hline
\end{tabular}

${ }^{a}$ Three standard reflns monitored every $3600 \mathrm{~s}$, intensity variation $<2 \%$, solvent: $\mathrm{CH}_{2} \mathrm{Cl}_{2}$ (disorder) for $\left[\mathrm{P}(2-\mathrm{py})_{3} \mathrm{Mo}(\mathrm{CO})_{3}\right] \cdot \mathrm{CH}_{2} \mathrm{Cl}_{2}$. Anion: $2 \mathrm{BF}_{4}^{-}$, Solvent: $\mathrm{CH}_{3} \mathrm{NO}_{2}$ for $\left[\mathrm{P}(2-\mathrm{py})_{3} \mathrm{Mo}\left(\mathrm{CH}_{3} \mathrm{CH}_{2} \mathrm{CN}\right)(\mathrm{NO})_{2}\right]-$ $\left(\mathrm{BF}_{4}\right)_{2} \cdot \mathrm{CH}_{3} \mathrm{NO}_{2}$.

${ }^{\mathrm{b}} R_{f}=\Sigma\left(F_{\mathrm{o}}-F_{\mathrm{c}}\right) / \Sigma\left(F_{\mathrm{o}}\right) ; R_{w}=\left[\Sigma\left(w\left(F_{\mathrm{o}}-F_{\mathrm{c}}\right)^{2}\right) / \Sigma\left(w F_{\mathrm{o}}^{2}\right)\right]^{1 / 2}$

${ }^{\mathrm{c}}$ Goodness of-fit $\left[\Sigma\left(w\left(F_{\mathrm{o}}-F_{\mathrm{c}}\right)^{2}\right) /(\right.$ number of reflections-number of parameters) $]^{1 / 2}$. 
Table 2

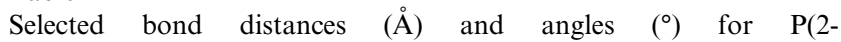
$\mathrm{Py})_{3} \mathrm{Mo}\left(\mathrm{CO}_{3}\right) \cdot \mathrm{CH}_{2} \mathrm{Cl}_{2}$

Bond distances
Mo-N(1)

$\mathrm{Mo}-\mathrm{N}(3)$

$2.279(3)$

$2.266(3)$

$1.928(4)$

$1.158(4)$

$\mathrm{C}(1)-\mathrm{O}(1)$

$\mathrm{C}(3)-\mathrm{O}(3)$

$1.165(4)$

Bond angles

$\mathrm{N}(1)-\mathrm{Mo}-\mathrm{N}(2)$

$\mathrm{N}(2)-\mathrm{Mo}-\mathrm{N}(3)$

$\mathrm{N}(1)-\mathrm{Mo}-\mathrm{C}(2)$

$\mathrm{N}(2)-\mathrm{Mo}-\mathrm{C}(3)$

$\mathrm{N}(3)-\mathrm{Mo}-\mathrm{C}(3)$

$\mathrm{N}(2)-\mathrm{Mo}-\mathrm{C}(2)$

$\mathrm{C}(1)-\mathrm{Mo}-\mathrm{C}(2)$

$\mathrm{C}(2)-\mathrm{Mo}-\mathrm{C}(3)$

\section{$84.56(10)$}

83.74(10)

$92.84(12)$

$97.49(13)$

92.51(12)

177.35(12)

$86.32(15)$

85.13(15)

$\begin{array}{ll}\mathrm{Mo}-\mathrm{N}(2) & 2.282(3) \\ \mathrm{Mo}-\mathrm{C}(1) & 1.951(4) \\ \mathrm{Mo}-\mathrm{C}(3) & 1.938(4) \\ \mathrm{C}(2)-\mathrm{O}(2) & 1.170(4)\end{array}$

\section{$\mathrm{N}(1)-\mathrm{Mo}-\mathrm{N}(3)$}

$\mathrm{N}(1)-\mathrm{Mo}-\mathrm{C}(1)$

$\mathrm{N}(2)-\mathrm{Mo}-\mathrm{C}(1)$

$\mathrm{N}(3)-\mathrm{Mo}-\mathrm{C}(2)$

$\mathrm{N}(1)-\mathrm{Mo}-\mathrm{C}(3)$

$\mathrm{N}(3)-\mathrm{Mo}-\mathrm{C}(1)$

C(1)-Mo-C(3)

$84.12(10)$

95.89(12)

93.39(13)

$175.85(12)$

87.60(14)
96.56(12)

177.12(13)

ceeding difference Fourier syntheses. Hydrogen atoms were located and added to the structures, but their position were not refined.

\section{Results and discussion}

3.1. Synthesis and characterization of $P(2-p y)_{3} M(C O)_{3}$, where $M=C r(\mathbf{1 a}), M o(\mathbf{1 b})$ and $W(\mathbf{1 c})$

The reactions of $\mathrm{P}(2-\mathrm{py})_{3}$ and 1 equiv. of $\mathrm{M}(\mathrm{CO})_{3}(\mathrm{CHCN})_{3}$, where $\mathrm{M}=\mathrm{Cr}$ and $\mathrm{W}$, or $\mathrm{Mo}(\mathrm{CO})_{6}$ in $\mathrm{CH}_{3} \mathrm{CN}$ resulted in the formation of complexes $\mathrm{P}(2-\mathrm{py})_{3} \mathrm{M}(\mathrm{CO})_{3}(\mathbf{1 a}-\mathbf{c})$. The infrared spectra of $\mathbf{1 a}-\mathbf{c}$ showing two $v_{\mathrm{CO}}$ bands (1a: 1901, 1777; 1b: 1908, 1797, and 1c: $1899,1784 \mathrm{~cm}^{-1}$ ) suggested that complexes 1a-c held a $C_{3 v}$ symmetry. As compared with the $\mathrm{CO}$ stretching frequency for $\mathrm{CO}$ gas $\left(2143 \mathrm{~cm}^{-1}\right)$, the $v_{\mathrm{CO}}$ found for $1 \mathbf{a}-\mathbf{c}$ were lower in energy due to a certain degree of metal-to-ligand $\pi$-back bonding. Only one set of pyridyl protons (between $\delta 7.15-9.55 \mathrm{ppm}$ ) with a ratio of $1: 1: 1: 1$ in the ${ }^{1} \mathrm{H}-\mathrm{NMR}$ spectra of $\mathbf{1 a}-\mathbf{c}$ indicated a $\eta^{3}-N, N^{\prime}, N^{\prime \prime}-\mathrm{P}(2-\mathrm{py})_{3}$ coordination. Complexes $\mathbf{1 a}-\mathbf{c}$ gave only one phosphorus resonance in their ${ }^{31} \mathrm{P}-\mathrm{NMR}$ spectra. The phosphorus chemical shift at $\delta$ $-4.5 \mathrm{ppm}$ for $1 \mathrm{c}$ indicated no sign of $\mathrm{P}-\mathrm{W}$ interaction.

Transparent purple crystals of $\mathrm{P}(2-$ py) ${ }_{3} \mathrm{Mo}(\mathrm{CO})_{3} \cdot \mathrm{CH}_{2} \mathrm{Cl}_{2}$ suitable for $\mathrm{X}$-ray crystallographic analysis were obtained from the recrystallization of $\mathbf{1 b}$ in $\mathrm{CH}_{3} \mathrm{CN}+\mathrm{CH}_{2} \mathrm{Cl}_{2}$ solution. The ORTEP drawing of $\mathrm{P}(2-\mathrm{py})_{3} \mathrm{Mo}(\mathrm{CO})_{3} \cdot \mathrm{CH}_{2} \mathrm{Cl}_{2}$ (Fig. 1) shows an almost perfect octahedral arrangement around the Mo center, and the $\mathrm{P}(2-\mathrm{py})_{3}$ ligand is occupying at the facial position of the octahedron through the coordination of three pyridyl nitrogen atoms with three N-Mo-C angles of $175.85,177.35$, and $177.12^{\circ}$ and three Mo-N bonds of 2.279, 2.282 and $2.266 \AA$ in length (Table 2).
3.2. Synthesis and characterization of

$\left[P(2-p y)_{3} M(C O)_{2}(N O)\right]\left(B F_{4}\right)$, where $M=M o(2 a)$ and $W(\boldsymbol{2 b})$

The reaction of $\mathrm{NO}^{+}$with low-valent transitionmetal complexes has been widely employed in the synthesis of nitrosyl complexes of both molybdenum and tungsten [16,17]. The treatment of $\mathbf{1 a}$ and $\mathbf{1 b}$ with 1 equiv. of $\mathrm{NOBF}_{4}$ in $\mathrm{CH}_{2} \mathrm{Cl}_{2}$ resulted in immediate gas evolution to afford complexes $\mathbf{2 a}$ and $\mathbf{2 b}$. The ${ }^{1} \mathrm{H}-\mathrm{NMR}$ spectra of both 2a and $\mathbf{2 b}$ showing two sets of pyridyl protons with a ratio of $2: 1$ indicated that the $C_{3 v}$ symmetry was no longer present. The higher CO stretching frequencies (2a: 2046, 1940 and 2b: 2012, $1912 \mathrm{~cm}^{-1}$ ) than those found for complexes $\mathbf{1 a}-\mathbf{c}$ suggested a weaker $\mathrm{M}-\mathrm{CO}$ interaction. However, complexes $\mathbf{2 a}$ and $\mathbf{2 b}$ still possessed rather high stability against further $\mathrm{CO}$ ligand replacement in most coordinating solvent systems. In addition to the $\mathrm{CO}$ stretching bands, the strong NO stretching bands at 1668 and $1650 \mathrm{~cm}^{-1}$ for $\mathbf{2 a}$ and $\mathbf{2 b}$ respectively were typical for linear $\mathrm{M}-\mathrm{N}-\mathrm{O}$ bonding mode [18].

\subsection{Synthesis and characterization of}

$\left[P(2-p y)_{3} M(C O)(N O)_{2}\right]\left(B F_{4}\right)_{2}$, where $M=M o$ (3a) and $W(3 \boldsymbol{b})$

Subsequent substitution of the second CO ligand can be achieved by treating complexes $\mathbf{2 a}$ and $\mathbf{2 b}$ with a further 1 equiv. of $\mathrm{NOBF}_{4}$ in $\mathrm{CH}_{3} \mathrm{NO}$. Alternatively, complexes $\mathbf{3 a}$ and $\mathbf{3 b}$ can also be synthesized by the direct reaction of $\mathbf{1 a}-\mathbf{b}$ and 2 equiv. of $\mathrm{NOBF}_{4}$ in $\mathrm{CH}_{3} \mathrm{NO}_{2}$. The ${ }^{1} \mathrm{H}-\mathrm{NMR}$ spectra of both $\mathbf{3 a}$ and $\mathbf{3 b}$ showing two sets of pyridyl protons with a ratio of 2:1 indicated that the $C_{3 v}$ symmetry was no longer present.

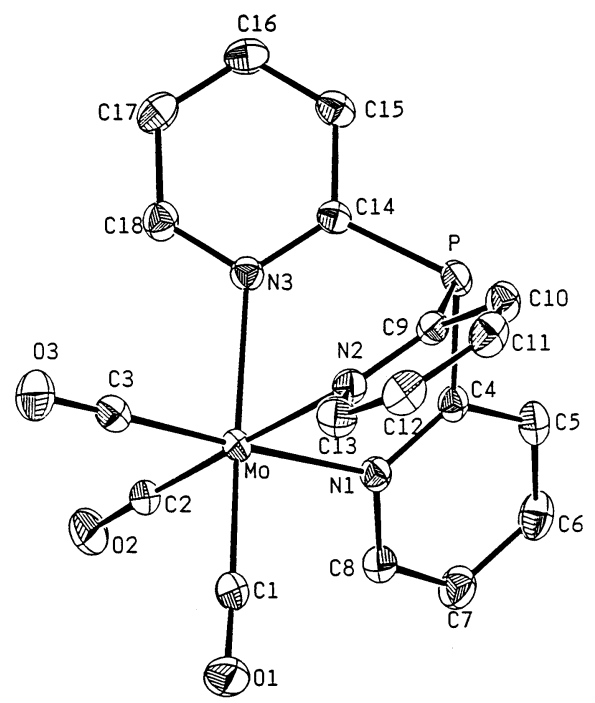

Fig. 1. ORTEP drawing of $\mathrm{P}(2-\mathrm{py})_{3} \mathrm{Mo}(\mathrm{CO})_{3} \cdot \mathrm{CH}_{2} \mathrm{Cl}_{2}$ showing the atom-labeling scheme used. Note that the $\mathrm{CH}_{2} \mathrm{Cl}_{2}$ molecule has been omitted for clarity. 
The only infrared $v_{\mathrm{CO}}$ band at 2037 and $2148 \mathrm{~cm}^{-1}$ for $\mathbf{3 a}$ and $\mathbf{3 b}$, respectively, was very close to that of free $\mathrm{CO}$, and was higher in energy than those found for 1a, 1b, 2a, and 2b. Apparently, these high CO stretching frequencies were attributed to the coordination of two $\mathrm{NO}^{+}$ligands. Thus, an expected weak $\mathrm{M}-\mathrm{CO}$ interaction could facilitate the loss of $\mathrm{CO}$ in the presence of a suitable coordinating ligand. In addition to the $\mathrm{CO}$ stretching bands, two strong NO stretching bands at $1839,1738 \mathrm{~cm}^{-1}$ and $1844,1785 \mathrm{~cm}^{-1}$ for $\mathbf{3 a}$ and $\mathbf{3 b}$, respectively, were typical for cis dinitrosyl complexes with linear $\mathrm{M}-\mathrm{N}-\mathrm{O}$ bonding modes [17,18]. As implied in the infrared spectral data, complexes $\mathbf{3 a}$ and $\mathbf{3 b}$ possessed a characteristic feature of strong $\mathrm{CO}$ lability when dissolving in donor solvents. Presumably, the loss of $\mathrm{CO}$ generated a 16-electron species, [P(2py) $\left.{ }_{3} \mathrm{M}(\mathrm{NO})_{2}\right]^{2+}$; the metal-anion interaction, therefore, became possible without the presence of coordinating solvent. Many examples of organometallic complexes of weakly coordinating anions, e.g. $\mathrm{BF}_{4}^{-}$, $\mathrm{PF}_{6}^{-}, \mathrm{AsF}_{6}^{-}$and $\mathrm{SbF}_{6}^{-}$, have been reported [19,20]. However, the infrared absorption bands of $v_{\mathrm{BF}}$ at 1026 and $1030 \mathrm{~cm}^{-1}$ for $\mathbf{3 a}$ and $\mathbf{3 b}$, respectively, indicated no asymmetric broadening of the $\mathrm{BF}_{4}^{-}$. Moreover, subsequent ${ }^{19} \mathrm{~F}-\mathrm{NMR}$ studies for $\mathbf{3 b}$ showing two equivalent $\mathrm{BF}_{4}^{-}$anions $(\delta-151.7 \mathrm{ppm})$ at room temperature suggested no direct $\mathrm{M}-\mathrm{F}-\mathrm{BF}_{3}$ interaction [10]. Apparently, the hard $\mathrm{BF}_{4}^{-}$anions in $\mathbf{3 a}$ and $\mathbf{3 b}$ did not bind to soft metal centers in the presence of coordinating solvents.

The CO lability in complexes $\mathbf{3 a}$ and $\mathbf{3 b}$ enables the isolation of a series of complexes, $[\mathrm{P}(2-$ py) $\left.{ }_{3} \mathrm{M}(\mathrm{L})(\mathrm{NO})_{2}\right]\left(\mathrm{BF}_{4}\right)_{2}(\mathrm{~L}=$ nitriles, aldehydes $)$. At ambient temperature, the facile $\mathrm{CO}$ loss of $\mathbf{3 a}$ and $\mathbf{3 b}$ in the presence of nitriles resulted in the formation of complexes $\left[\mathrm{P}(2-\mathrm{py})_{3} \mathrm{M}\left(\mathrm{CH}_{3} \mathrm{CN}\right)(\mathrm{NO})_{2}\right]\left(\mathrm{BF}_{4}\right)_{2}(\mathbf{4 a}, \mathrm{M}=\mathrm{Mo}$; 5a, $\mathrm{M}=\mathrm{W})$ and $\left[\mathrm{P}(2-\mathrm{py})_{3} \mathrm{M}\left(\mathrm{CH}_{3} \mathrm{CH}_{2} \mathrm{CN}\right)(\mathrm{NO})_{2}\right]\left(\mathrm{BF}_{4}\right)_{2}$ $(\mathbf{4 b}, \mathrm{M}=\mathrm{Mo} ; \mathbf{5 b}, \mathrm{M}=\mathrm{W})$. A total conversion of $\mathbf{4 a}$ and $\mathbf{5 a}$ to $\mathbf{4 b}$ and $\mathbf{5 b}$ can be accomplished within hours in the presence of a stoichiometric amount of $\mathrm{CH}_{3} \mathrm{CN}$. Transparent green crystals of $\left[\mathrm{P}(2-\mathrm{py})_{3} \mathrm{Mo}\left(\mathrm{CH}_{3}-\right.\right.$ $\left.\left.\mathrm{CH}_{2} \mathrm{CN}\right)(\mathrm{NO})_{2}\right]\left(\mathrm{BF}_{4}\right)_{2} \cdot \mathrm{CH}_{3} \mathrm{NO}_{2}$ suitable for X-ray crystallographic analysis were obtained from the recrystallization of $3 \mathrm{a}$ in $\mathrm{CH}_{3} \mathrm{CH}_{2} \mathrm{CN} / \mathrm{CH}_{3} \mathrm{NO}_{2}$ (1:1) solutions. The ORTEP drawing of $\left[\mathrm{P}(2-\mathrm{py})_{3} \mathrm{Mo}\left(\mathrm{CH}_{3} \mathrm{CH}_{2} \mathrm{CN}\right)-\right.$ $\left.(\mathrm{NO})_{2}\right]\left(\mathrm{BF}_{4}\right)_{2} \cdot \mathrm{CH}_{3} \mathrm{NO}_{2}$ (Fig. 2) shows an almost perfect octahedral arrangement around the Mo center, and the $\mathrm{P}(2-\mathrm{py})_{3}$ ligand occupies the facial position of the octahedron through the coordination of three pyridyl nitrogen atoms. The almost linear $\mathrm{Mo}-\mathrm{N}-\mathrm{O}$ arrangement with the angles $\mathrm{M}-\mathrm{N}-\mathrm{O}$ of 175.0 and $177.0^{\circ}$ (Table 3) indicates the two $\mathrm{NO}^{+}$ligands are $c$ is to each other.

A similar chemistry has also been observed when $\mathbf{3 a}$ and $\mathbf{3 b}$ were treated with Lewis basic organic carbonyl compounds. In the presence of excess amounts of alde-

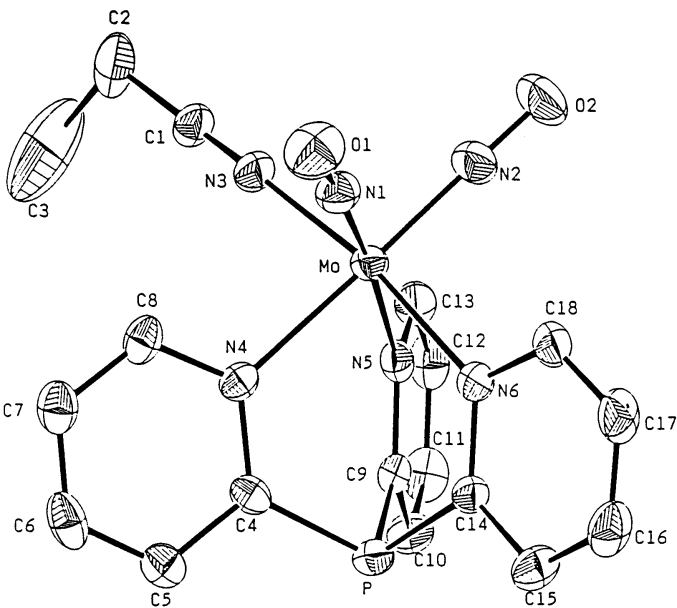

Fig. 2. ORTEP drawing of $\left[\mathrm{P}(2-\text { py })_{3} \mathrm{Mo}\left(\mathrm{CH}_{3} \mathrm{CH}_{2} \mathrm{CN}\right)-\right.$ $\left.(\mathrm{NO})_{2}\right]\left(\mathrm{BF}_{4}\right)_{2} \cdot \mathrm{CH}_{3} \mathrm{NO}_{2}$ showing the atom-labeling scheme used. Note that $\mathrm{CH}_{3} \mathrm{NO}_{2}$ and $\mathrm{BF}_{4}^{-}$have been omitted for clarity.

hydes, the $\left[\mathrm{P}(2-\mathrm{py})_{3} \mathrm{M}(\mathrm{NO})_{2}\right]\left(\mathrm{BF}_{4}\right)_{2}-$ crotonaldehyde adducts $4 \mathbf{c}(\mathrm{M}=\mathrm{Mo})$ and $\mathbf{5 c},(\mathrm{M}=\mathrm{W})$ as well as $[\mathrm{P}(2-$ py $\left.)_{3} \mathrm{~W}(\mathrm{NO})_{2}\right]\left(\mathrm{BF}_{4}\right)_{2}$-benzaldehyde adduct $\mathbf{5 d}$ can be spectroscopically analyzed by NMR. Attempts to isolate these aldehyde adducts failed, probably because the relative basicities of these aldehydes were not strong enough, as can be seen in the isolated $[\mathrm{P}(2-$ py) $\left.{ }_{3} \mathrm{M}(\mathrm{NO})_{2}\right]\left(\mathrm{BF}_{4}\right)_{2}-$ nitrile adducts $4 \mathbf{a}-\mathbf{b}$ and $\mathbf{5 a}-\mathbf{b}$.

\subsection{Lewis acidity and reactivity of $[P(2-$ py) $\left.)_{3} W(\mathrm{CO})(\mathrm{NO})_{2}\right]\left(\mathrm{BF}_{4}\right)_{2}(\mathbf{3 b})$}

Organometallic Lewis acids are of great importance in the catalysis of carbon-carbon bond formation reactions because the steric bulk of the supporting ligands

Table 3

Selected bond distances $(\AA)$ and angles $\left(^{\circ}\right)$ for $[\mathrm{P}(2$ py) $\left.{ }_{3} \mathrm{Mo}\left(\mathrm{CH}_{3} \mathrm{CH}_{2} \mathrm{CN}\right)(\mathrm{NO})_{2}\right]\left(\mathrm{BF}_{4}\right)_{2} \cdot \mathrm{CH}_{3} \mathrm{NO}_{2}$

$\begin{array}{lrlr}\text { Bond distances } & & & \\ \text { Mo-N(1) } & 1.820(9) & \text { Mo-N(2) } & 1.819(9) \\ \text { Mo-N(3) } & 2.149(8) & \text { Mo-N(4) } & 2.202(9) \\ \text { Mo-N(5) } & 2.206(8) & \text { Mo-N(6) } & 2.160(8) \\ \mathrm{N}(1)-\mathrm{O}(1) & 1.155(12) & \mathrm{N}(2)-\mathrm{O}(2) & 1.147(13) \\ \mathrm{N}(3)-\mathrm{C}(1) & 1.112(13) & \mathrm{C}(1)-\mathrm{C}(2) & 1.464(16) \\ \mathrm{C}(2)-\mathrm{C}(3) & 1.59(3) & & \\ \text { Bond angles } & & & \\ \mathrm{N}(1)-\mathrm{Mo}-\mathrm{N}(2) & 90.6(5) & \mathrm{N}(1)-\mathrm{Mo}-\mathrm{N}(3) & 94.3(4) \\ \mathrm{N}(1)-\mathrm{Mo}-\mathrm{N}(4) & 89.1(4) & \mathrm{N}(1)-\mathrm{Mo}-\mathrm{N}(5) & 175.0(4) \\ \mathrm{N}(1)-\mathrm{Mo}-\mathrm{N}(6) & 93.1(3) & \mathrm{N}(2)-\mathrm{Mo}-\mathrm{N}(3) & 92.9(4) \\ \mathrm{N}(2)-\mathrm{Mo}-\mathrm{N}(4) & 178.1(3) & \mathrm{N}(2)-\mathrm{Mo}-\mathrm{N}(5) & 94.4(4) \\ \mathrm{N}(2)-\mathrm{Mo}-\mathrm{N}(6) & 94.0(4) & \mathrm{N}(3)-\mathrm{Mo}-\mathrm{N}(4) & 85.2(3) \\ \mathrm{N}(3)-\mathrm{Mo}-\mathrm{N}(5) & 85.8(3) & \mathrm{N}(3)-\mathrm{Mo}-\mathrm{N}(6) & 169.8(3) \\ \mathrm{N}(4)-\mathrm{Mo}-\mathrm{N}(5) & 85.9(3) & \mathrm{N}(4)-\mathrm{Mo}-\mathrm{N}(6) & 87.9(3) \\ \mathrm{N}(5)-\mathrm{Mo}-\mathrm{N}(6) & 86.2(3) & \mathrm{Mo}-\mathrm{N}(1)-\mathrm{O}(1) & 177.0(10) \\ \mathrm{Mo}-\mathrm{N}(2)-\mathrm{O}(2) & 178.5(9) & \mathrm{Mo}-\mathrm{N}(3)-\mathrm{C}(1) & 178.5(9)\end{array}$



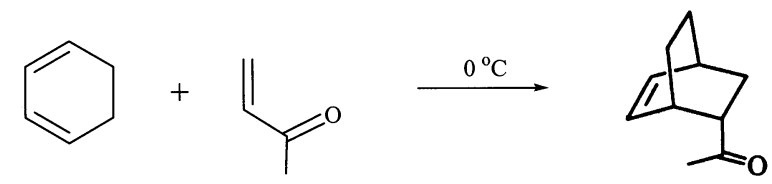

\begin{tabular}{|cccccc|}
\hline $\begin{array}{c}\text { Catalyst loading } \\
\text { (3b, mol \%) }\end{array}$ & Solvent & $\begin{array}{c}\text { Time } \\
\text { (hrs) }\end{array}$ & endo : exo & \% conversion & $\begin{array}{c}\text { Yield } \\
\text { (\%) }\end{array}$ \\
\hline 0.3 & $\mathrm{CH}_{2} \mathrm{Cl}_{2}$ & 24 & $99: 1$ & 95 & 85 \\
0.3 & $\mathrm{CH}_{3} \mathrm{NO}_{2}$ & 15 & $99: 1$ & 93 & 81 \\
\hline
\end{tabular}

Scheme 1.

and the electronic character of the metals can offer influential effects on the rate and selectivity. The Lewis acidities of complexes $\mathbf{3 a}$ and $\mathbf{3 b}$ are reflected in their facile formation of nitrile $(\mathbf{4 a}, \mathbf{4 b}, \mathbf{5 a}, \mathbf{5 b})$ and aldehyde adducts $(\mathbf{4 c}, \mathbf{5 c}, \mathbf{5 d})$. The method developed by Childs et al. [21a] and Hersh and co-workers [21b] took the ${ }^{1} \mathrm{H}-\mathrm{NMR}$ chemical shift differences of the 3-position hydrogen of crotonaldehyde before and after coordinated to a Lewis acid as a relative scale to determine the acid strength. In our system, the complexation of crotonaldehyde provided downfield shifts of $\Delta \delta 1.01$ $\mathrm{ppm}$ and $\Delta \delta 1.18 \mathrm{ppm}$ for $\mathbf{3} \mathbf{a}$ and $\mathbf{3 b}$, respectively, and the acidities of $\mathbf{3 a}$ and $\mathbf{3 b}$ were found to be comparable to those of $\mathrm{TiCl}_{4}(\Delta \delta 1.03 \mathrm{ppm})$ and $\mathrm{BF}_{3}(\Delta \delta 1.17 \mathrm{ppm})$ and $\mathrm{AlCl}_{3}(\Delta \delta 1.23 \mathrm{ppm})$, respectively. Accordingly, we looked into the study of Lewis acid reactivity of complex 3b for the classical Diels-Alder catalysis. As indicated in Scheme 1, complex $\mathbf{3 b}$ has shown potential activity in promoting the cycloaddition reaction of cyclohexadiene and methyl vinyl ketone. With a loading of $0.3 \mathrm{~mol} \%$ of $\mathbf{3 b}$, the cycloaddition reactions provided an average of $94 \%$ conversion with a $99 \%$ endo-selectivity, while the corresponding thermal reaction only afforded a less than $5 \%$ conversion at $0^{\circ} \mathrm{C}$. An average $83 \%$ isolated yield of the Diels-Alder adduct was obtained when the reaction mixtures were purified by column chromatography.

The polydiene is often observed as a potential sideproduct of Lewis acid-catalyzed Diels-Alder reactions. However, at a reaction temperature of $0^{\circ} \mathrm{C}$, no polymeric materials were observed in the NMR spectroscopic analysis nor insoluble precipitates formed during the course of 3b-catalyzed Diels-Alder reactions of cyclohexadiene and methyl vinyl ketone. The reactivities, kinetic and mechanistic insights of the catalysis will be subjected to further investigation.

\section{Supplementary information}

Tables of crystal data, complete bond angles and distances, atomic coordinates, and equivalent displacement coefficients for $\mathrm{P}(2-\mathrm{py})_{3} \mathrm{Mo}(\mathrm{CO})_{3} \cdot \mathrm{CH}_{2} \mathrm{Cl}_{2}$ (17 pages) and $\left[\mathrm{P}(2-\mathrm{py})_{3} \mathrm{Mo}\left(\mathrm{CH}_{3} \mathrm{CH}_{2} \mathrm{CN}\right)(\mathrm{NO})_{2}\right]\left(\mathrm{BF}_{4}\right)_{2}$.
$\mathrm{CH}_{3} \mathrm{NO}_{2}$ (23 pages). Ordering information is given on any current masthead page.

\section{Acknowledgements}

Financial supports from the National Science Council (NSC88-2113-M-194-018) and National Chung Cheng University are gratefully acknowledged.

\section{References}

[1] W.C. Davies, F.G. Mann, J. Chem. Soc. (1944) 276.

[2] J.E. Parker, B.E. Wagner, R.H. Holm, J. Am. Chem. Soc. 92 (1970) 3500.

[3] C.J.L. Lock, M.A. Turner, Acta. Cryst. Sect. C 43 (1987) 2096.

[4] Y. Xie, B.R. James, J. Organomet. Chem. 417 (1991) 277.

[5] (a) Y. Xie, C.-L. Lee, Y. Yang, S.J. Rettig, B.R. James, Can. J. Chem. 70 (1992) 751. (b) R.P. Schutte. Ph.D. Dissertation, University of British Columbia, 1995.

[6] (a) K. Kurtev, D. Ribola, R.A. Jones, D.J. Cole-Hamulton and G.J. Wilkinson, J. Chem. Soc. Dalton Trans. (1980) 55. (b) I.R. baird, M.B. Smith, B.R. James, Inorg. Chim. Acta. 235 (1995) 291, and references therein.

[7] (a) A.J. Deeming, M.B. Smith, J. Chem. Soc. Chem. Commun. (1993) 844. (b) L.Y. Xie, B.R. James, Inorg. Chim. Acta. 217 (1994) 209.

[8] (a) R.K. Boggess, D.A. Zatko, J. Coord. Chem. 4 (1975) 217. (b) F.R. Keene, M.R. Snow, P.J. Stephenson, E.R.T. Tiekink, Inorg. Chem. 27 (1988) 2040. (c) P.S. Moritz, A.A. Diamantis, F.R. Keene, M.R. Snow, E.R. T. Tiekink, Aust. J. Chem. 41 (1988) 1353. (d) R. Gregorzik, J. Wirbser, H. Vahrenkamp, Chem. Ber. 125 (1992) 1575. (e) R.P. Schutte, S.J. Rettig, B. R. James, Can. J. Chem. 74 (1996) 2064.

[9] (a) R.K. Boggess, D.A. Zatko, Inorg. Nucl. Chem. Lett. 12 (1976) 7. (b) R.K. Boggess, D.A. Zatko, Inorg. Chem. 15 (1976) 626. (c) R.A. Kenneth, P.A. Anderson, T. Astley, I.M. Atkinson, J.M. Charnock, C.D. Garner, J.M. Gulbis, T.W. Hambley, M.A. Hitchman, F.R. Keene, E.R.T. Tiekink, J. Chem. Soc. Dalton Trans. (1997) 519.

[10] J.W. Faller, Y. Ma, J. Am. Chem. Soc. 113 (1991) 1579.

[11] J.W. Faller, L.-L. Gundersen, Tetrahedron Lett. 34 (1993) 2275.

[12] (a) E. Lindner, H. Rauleder, P. Wegner, Z. Naturforsch. Teil B 39 (1984) 1224. (b) G.R. Newkome, Chem. Rev. 93 (1993) 2067, and references therein.

[13] D.P. Tate, W.R. Knipple, J.M. Augl, Inorg. Chem. 1 (1962) 433.

[14] For detailed references, see supplementary material of crystallographic analyses.

[15] E.J. Gabe, Y. Le Page, J.-P. Charland, F.L. Lee, P.S. White, J. Appl. Cryst. 22 (1989) 384.

[16] (a) M. Green, S.H. Taylor, J. Chem. Soc. Dalton, Trans. (1972) 2629. (b) A. Sen, R. Thomas, Organometallics 1 (1982) 1251.

[17] R.P. Stewart Jr., G.T. Moore, Inorg. Chem. 14 (1975) 2699.

[18] (a) J.H. enemark, R.D. Feltham, Coord. Chem. Rev., 13 (1974) 329. (b) T.W. Hawkins, M.B. Hall, Inorg. Chem. 19 (1980) 1735.

[19] W. Beck, K. Sünkel, Chem. Rev. 88 (1988) 1405, and references therein.

[20] (a) W.H. Hersh, J. Am. Chem. Soc. 107 (1985) 4599. (b) R.V. Honeychuck, P.V. Bonnesen, J. Farahi, W.H. Hersh, J. Org. Chem. 52 (1987) 5293. (c) P.V. Bonnesen, C.L. Puckett, R.V. Honeychuck, W.H. Hersh, J. Am. Chem. Soc. 111 (1989) 6070. (d) S. Sato, I. Matsuda, Y. Izumi, Terahedron Lett. 27 (1986) 5517.

[21] (a) R.F. Childs, D.L. Mulholland, A. Nixon, Can. J. Chem. 60 (1982) 801. (b) P.V. Bonnesen, C.L. Puckett, R.V. Honeychuck, W.H. Hersh, J. Am. Chem. Soc. 111 (1989) 6070. 